\title{
A Study of the Relationship between Convex Sets and Connected Sets
}

\author{
Khem Raj Malla ${ }^{1} \&$ Prakash Muni Bajracharya ${ }^{2}$
}

${ }^{1} \mathrm{PhD}$ Scholar, Mewar University, Rajasthan, India

${ }^{2}$ Research Supervisor, Professor, Tribhuvan University, Kirtipur, Kathmandu, Nepal

\section{Corresponding Author}

Khem Raj Malla

Email: khemrajmalla12@gmail.com

\begin{abstract}
The principal objective of this research article is to explore the relationship between convex sets and connected sets. All convex sets are connected but in all cases connected sets are not convex. In the Maly theorem,let $X$ be a Banach space, and let $f: X \rightarrow R$ be a (Fr'echet-)differentiable function. Then, for any closed convex subset $C$ of $X$ with nonempty interior, the image $D f(C)$ of $C$ by the differential $D f$ of $f$ is a connected subset of $X *$, where $X *$ stands for thetopological dual space of $X$.The result does not hold true if $C$ has an empty interior. There are counterexamples even with functions $f$ of two variables. This article concludes that convexity cannot be replaced with the connectedness of $C$.
\end{abstract}

\section{KEYWORDS}

Connectedness, Convex sets, Function

\section{INTRODUCTION}

We have convexity as a local property of contour curvature: the local sign of curvature. A different use of the term refers, instead, to the global degree of convexity of complex 2-D shapes. The convex hull or envelope of a set of points or vertices of a polygon is defined as the minimal convex set containing all those points. However, very different shapes can share the same convex hull. Using the convex hull, we can define a global measure of shape convexity as the proportion of the area of a polygon over the area of its convex hull (Preparata and Shamos, 1985). More sophisticated measures of global convexity have been put forward, for instance, by Pao, Geiger, and Rubin (1999) and by Rosin (2000). So far, these measures have not been fully validated in terms of their role in human perception. It can clearly be useful to have a formula that assigns a single value of convexity to any complex 2-D shape. In image analysis, this type of measure is known as a shape factor: a value that is affected by an object's shape but is independent of its dimensions. An example that is closely related to that of convexity is that of compactness, defined as the square of the perimeter over the area or, alternatively, as the area of a shape over the area of a circle having the same perimeter. Another well-known shape factor is the aspect ratio.

In the previous paragraph, we contrasted a local meaning of convexity (the sign of curvature at one location along a contour) and a global meaning of convexity. An intermediate 
definition is also possible. For example, Fowlkes, Martin, and Malik (2007) computed convexity within a region from the fraction of point pairs for which a straight line connecting the two points lies completely within the region. This value can be computed for both sides of the contour, and convexity is then given by the log ratio of the two values. Changing the size of the region would make this measure more local or more global.

A figure is called convex if it contains, along with any pair of its points $x, y$, also the entire segment $[x, y]$ joining the points. This is exactly the definition of a convex set in the multidimensional case; all we need is to say what does it mean "the segment $[x, y]$ joining the points $x, y \in \mathrm{R}^{n}$ "(Lecture1https://ljk.imag.fr/membres/Anatoli.Iouditski/cours /convex/chapitre1.pdf). A subset $\mathrm{M}$ of $\mathrm{R}^{\mathrm{n}}$ is called convex, if it contains, along with any pair of its points $x, y$, also the entire segment $[x, y]: x, y \in M, 0 \leq \lambda \leq 1 \Rightarrow \lambda x+(1-\lambda) y \in M$.

In geometry, a convex set or a convex region is a subset of a Euclidean space, or more generally an affine space over the reals, that intersects every line into a line segment (Morris and Stark, 2017 and Kjeldsen, 2010).Equivalently, this is a subset that is closed under convex combinations ( Bachem and Kern, 2017). For example, a solid cube is a convex set, but anything that is hollow or has an indent, for example, a crescent shape, is not convex.

The boundary of a convex set is always a convex curve. The intersection of all the convex sets that contain a given subset $A$ of Euclidean space is called the convex hull of $A$. It is the smallest convex set containing $A$.

A convex function is a real-valued function defined on an interval with the property that its epigraph (the set of points on or above the graph of the function) is a convex set. Convex minimization is a subfield of optimization that studies the problem of minimizing convex functions over convex sets. The branch of mathematics in which we study the properties of convex sets and convex functions is called convex analysis.

\section{PRELIMINARIES}

Segment:Let $\mathrm{x}$, $\mathrm{y}$ be two points in $\mathbf{R}^{\mathrm{n}}$. The set

$$
[x, y]=\{z=\lambda x+(1-\lambda) y \mid 0 \leq \lambda \leq 1\}
$$

is called a segment with the endpoints $\mathrm{x}, \mathrm{y}$.

Convex Set: A subset $\mathrm{M}$ of $\mathbf{R}^{\mathrm{n}}$ is called convex, if it contains, along with any pair of its points $\mathrm{x}$, $\mathrm{y}$,also the entire segment $[\mathrm{x}, \mathrm{y}]$ :

$$
x, y \in M, 0 \leq \lambda \leq 1 \Rightarrow \lambda x+(1-\lambda) y \in M .
$$

Note that by this definition an empty set is convex (by convention, or better to say, by the exact sense of the definition: for the empty set, we cannot present a counterexample to show that it is not convex).

The simplest examples of nonempty convex sets are singletons - points - and the entire space $\mathbf{R}^{n}$. A much more interesting example is as follows:

Example: The solution set of an arbitrary (possibly, infinite) system

$\mathrm{a}_{\alpha}^{\mathrm{T}} \mathrm{x} \leq \mathrm{b}_{\alpha}, \alpha \in \mathrm{A}$

of linear inequalities with $\mathrm{n}$ unknowns $\mathrm{x}-$ the set

$$
\mathrm{M}=\left\{\mathrm{x} \in \mathbf{R}^{\mathrm{n}} \mid \mathrm{a}_{\mathrm{\alpha}}^{\mathrm{T}} \mathrm{x} \leq \mathrm{b}_{\alpha}, \alpha \in A\right\}
$$

is convex. 
In particular, the solution set of a finite system

$$
\mathrm{Ax} \leq \mathrm{b}
$$

of $\mathrm{m}$ inequalities with $\mathrm{n}$ variables ( $\mathrm{A}$ is $\mathrm{m} \times \mathrm{n}$ matrix) is convex; a set of this latter type is called polyhedral.

Indeed, let $x, y$ be two solutions to the system; we should prove that any point $z=\lambda x+(1-$ $\lambda y)$ with $\lambda \in[0,1]$ also is a solution to the system. This is evident, since for every $\alpha \in A$ we have

$$
\begin{aligned}
& a_{\alpha}^{T} x \leq b_{\alpha} \\
& a_{\alpha}^{T} y \leq b_{\alpha}
\end{aligned}
$$

whence, multiplying the inequalities by nonnegative reals $\lambda$ and $1-\lambda$ and taking sum of the results,

$$
\lambda a_{\kappa}^{T} x+(1-\lambda) a_{\kappa}^{T} y \leq \lambda b_{\alpha}+(1-\lambda) b_{\kappa}=b_{\alpha}
$$

and what is in the left hand side is exactly $a_{\approx}^{T} z$.

\section{Remark}

Note that any set given by Example 2.2.1 is not only convex, but also closed.

Any plane in $\mathbf{R}^{\mathrm{n}}$ (in particular, any linear subspace) is the set of all solutions to some system of linear equations. Now, a system of linear equations is equivalent to a system of linear inequalities (we can equivalently represent a linear equality by a pair of opposite linear inequalities). It follows that a plane is a particular case of a polyhedral set and is therefore convex. Of course, we could obtain this conclusion directly: convexity of a set means that it is closed with respect to taking certain restricted set of linear combinations of its members namely, the pair combinations with nonnegative coefficients of unit sum. We can show that any plane or an affine set is closed with respect to taking linear combinations not compulsory positive of its elements with unit sum.

\section{INNER DESCRIPTION OF CONVEX SETS: CONVEX COMBINATIONS AND CONVEX HULL}

Definition: A convex combination of vectors $y_{1}, \ldots, y_{m}$ is their linear combination

$$
y=\sum_{i=1}^{m} \lambda_{i} y_{i}
$$

with nonnegative coefficients with unit sum:

$$
\lambda_{\mathrm{i}} \geq 0, \sum_{\mathrm{i}=1}^{\mathrm{m}} \lambda_{\mathrm{i}}=1
$$

We have the following simple statement:

Proposition : A set $\mathrm{M}$ in $\mathbf{R}^{\mathrm{n}}$ is convex if and only if it is closed with respect to taking all convex combinations of its elements, i.e., if and only if any convex combination of vectors from $\mathrm{M}$ again is a vector from $\mathrm{M}$.

Proof.

Necessarypart: Assume that $M$ contains all convex combinations of the elements of $M$. Then,

Vol. 6. No. I

www.phdcentre.edu.np 
with any two points $x, y \in M$ and any $\lambda \in[0,1], M$ contains also the vector $\lambda x+(1-\lambda) y$, since it is a convex combination of $x$ and $y$; thus, $M$ is convex. Sufficient part: Assume that $M$ is convex; we should prove that then $M$ contains any convex combination.

$$
\text { (*) } \quad y=\sum_{i=1}^{m} \lambda_{i} y_{i}
$$

of vectors $y_{i} \in M$. The proof is given by induction in $m$. The case of $m=1$ is evident (since the only 1-term convex combinations are of the form is $1 \cdot y_{1}=y_{1} \in M$ ). Assume that we already know that any convex combination of $m-1$ vectors, $m \geq 2$, from $M$ is again a vector from $M$, and let us prove that this statement remains valid also for all convex combinations of $m$ vectors from $M$. Let $\left(^{*}\right)$ be such a combination. We can assume that $1>\lambda_{m}$, since otherwise there is nothing to prove (indeed, if $\lambda_{m}=1$, then the remaining $\lambda_{i}$ 's should be zero, since all $\lambda$ 's are nonnegative with the unit sum, and we have $\mathrm{y}=\mathrm{y}_{m} \in \mathrm{M}$ ).

Assuming $\lambda_{m}<1$, we can write

$$
y=\left(1-\lambda_{m}\right)\left[\sum_{i=1}^{m-1} \frac{\lambda_{i}}{1-\lambda_{m}} y_{i}\right]+\lambda_{m} y_{m} .
$$

The value in the brackets is a convex combination of $m-1$ points from $M$ and therefore, by the inductive hypothesis, this is a point, let it be called $z$, from $M$; we have

$$
y=\left(1-\lambda_{m}\right) z+\lambda_{m} y_{m}
$$

with $z$ and $y_{m} \in M$, and $y \in M$ by definition of a convex set $M$.

\section{Convex hull}

Proposition [Convexity of intersections] Let $\left\{\mathrm{M}_{\alpha}\right\}_{\alpha}$ be an arbitrary family of convex subsets of $\mathbf{R}^{\mathrm{n}}$. Then the intersection

$$
\mathrm{M}=\cap_{\alpha} \mathrm{M}_{\alpha}
$$

is convex.

Indeed, if the endpoints of a segment $[x, y]$ belong to $M$, then they belong also to every $M_{\alpha}$; due to the convexity of $M_{\alpha}$, the segment $[x, y]$ itself belongs to every $M_{\alpha}$, and, consequently, to their intersection, i.e., to $M$.

An immediate consequence of this Proposition is as follows:

Corollary [Convex hull]

Let $\mathrm{M}$ be a nonempty subset in $\mathbf{R}^{\mathrm{n}}$. Then among all convex sets containing $\mathrm{M}$ (these setsexist, e.g., $\mathbf{R}^{\mathrm{n}}$ itself) there exists the smallest one, namely, the intersection of all convex setscontaining M.This set is called the convex hull of M.

Proposition [Convex hull via convex combinations] For a nonempty $\mathrm{M} \subset \mathbf{R}^{\mathrm{n}}$ : $\operatorname{Conv}(\mathrm{M})=\{$ the set of all convex combinations of vectors from $\mathrm{M}\}$.

Proof. Any convex set containing $M$ (in particular, Conv $(M)$ ) contains all convex combinations of vectors from $M$. What remains to prove is that $\operatorname{Conv}(M)$ does not contain anything else. To this end it suffices to prove that the set of all convex combinations of vectors from $M$, let this set be called $M *$, itself is convex (given this fact and taking into account that Conv $(M)$ is the smallest convex set containing $M$, we achieve our goal - the inclusion Conv $(M) \subset M *)$. To prove that $M *$ is convex is the same as to prove that any convex combination $v x+(1-v) y$ of any two points $x=\sum_{i} \lambda_{i} x_{i}, y=\sum_{i} \mu_{i} x_{i}$ of $M^{*}-$ two convex combinations of vectors $\mathrm{x}_{i} \in \mathrm{M}$ is 
ISSN: 2362-1303 (Paper) | eISSN: 2362-1311 (Online)

JOURNAL OF ADVANCED ACADEMIC RESEARCH (JAAR)

again a convex combination of vectors from $M$. This is evident:

$v x+(1-v) y=v \sum_{i} \lambda_{i} x_{i}+(1-v) \sum_{i} \mu_{i} x_{i}=\sum_{i} \xi_{i} x_{i}, \xi_{i}=v \lambda_{i}+(1-v) \mu_{i}$,

and the coefficients $\xi_{i}$ clearly are nonnegative with unit sum.

\section{MORE EXAMPLES OF CONVEX SETS: POLYTOPE AND CONE}

A polytope is, by definition, the convex hull of a finite nonempty set in $\mathbf{R}^{n}$, i.e., the set of the form

$\operatorname{Conv}\left(\left\{u_{1}, \ldots, u_{N}\right\}\right)=\left\{\sum_{i}^{N} \lambda_{i} u_{i}=\mid \lambda_{i} \geq 0, \sum_{i} \lambda_{i}=1\right\}$.

An important case of a polytope is a simplex - the convex hull of $n+1$ affinely independent points $v_{1}, \ldots, v_{n+1}$ from $\mathbf{R}^{n}$ :

$$
M=\operatorname{Conv}\left(\left\{v_{1}, \ldots, v_{n+1}\right\}\right)=\left\{\sum_{i}^{n+1} \lambda_{i} v_{i} \mid \lambda i \geq 0, \sum_{i}^{n+1} \lambda_{i}=1\right\}
$$

the points $v_{1}, \ldots, v_{n+1}$ are called vertices of the simplex.

A cone.A nonempty subset $M$ of $\mathbf{R}^{n}$ is called conic, if it contains, along with every point $x \in M$, the entire ray $R x=\{t x \mid t \geq 0\}$ spanned by the point:

$x \in M \Rightarrow t x \in M \forall t \geq 0$.

A convex conic set is called a cone.

Proposition 4.2.1.A nonempty subset $\mathrm{M}$ of $\mathrm{R}^{\mathrm{n}}$ is a cone if and only if it possesses the

following pair of properties:

- is conic: $x \in M, t \geq 0 \Rightarrow t x \in M$;

- contains sums of its elements: $x, y \in M \Rightarrow x+y \in M$.

As an immediate consequence, we get that a cone is closed with respect to taking linear combinations with nonnegative coefficients of the elements, and vice versa - a nonempty set closed with respect to taking these combinations is a cone.

Example 4.2.2 The solution set of an arbitrary (possibly, infinite) system

$\mathrm{a}_{\alpha}^{\mathrm{T}} \mathrm{x} \leq \mathrm{b}_{\alpha}, \alpha \in \mathrm{A}$

of homogeneous linear inequalities with $\mathrm{n}$ unknowns $\mathrm{x}$ - the set

$\mathrm{K}=\left\{\mathrm{x} \mid \mathrm{a}_{\alpha}^{\mathrm{T}} \mathrm{x} \leq 0, \alpha \in \forall \alpha \in \mathrm{A}\right\}$

is a cone.

In particular, the solution set to a homogeneous finite system of $\mathrm{m}$ homogeneous linear inequalities

$$
\text { Ax } \leq 0
$$

( $\mathrm{A}$ is $\mathrm{m} \times \mathrm{n}$ matrix) is a cone; a cone of this latter type is called polyhedral.

Cones form a very important family of convex sets, and one can develop theory of cones absolutely similar (and in a sense, equivalent) to that one of all convex sets.E.g., introducing the notion of conic combinationof vectors $x_{1}, \ldots, x_{k}$ as a linear combination of the vectors with nonnegative coefficients, you can easily prove the following statements completely similar to those for general convex sets, with conic combination playing the role of convex one:

- A set is a cone if and only if it is nonempty and is closed with respect to taking all conic combinations of its elements; 
- Intersection of any family of cones is again a cone; in particular, for any nonempty set $M$ $\subset \mathbf{R}^{n}$ there exists the smallest cone containing $M$ - its conic hull Cone $(M)$, and this conic hull is comprised of all conic combinations of vectors from $M$. In particular, the conic hull of a nonempty finite set $M=\left\{u_{1}, \ldots, u_{N}\right\}$ of vectors in $\mathbf{R}^{n}$ is the cone

$$
\text { Cone }(M)=\left\{N \sum_{i=1}^{N} \lambda_{i} u_{i} \mid \lambda_{i} \geq 0, i=1, \ldots, N\right\} .
$$

A fundamental fact (cf. the above story about polytopes) is that this is the generic (inner) description of a polyhedral cone - of a set given by (outer description) finitely many homogeneous linear inequalities.

\section{ALGEBRAIC PROPERTIES OF CONVEX SETS}

The following statement is an immediate consequence of the definition of a convex set.

PropositionThe following operations preserve convexity of sets:

- Arithmetic summation and multiplication by reals: if $\mathrm{M}_{1}, \ldots, \mathrm{M}_{\mathrm{k}^{\prime}}$ are convex sets in $\mathbf{R}^{\mathrm{n}}$ and $\lambda_{1}, \ldots, \lambda_{\mathrm{k}}$ are arbitrary reals, then the set

$\lambda_{1} M_{1}+\ldots+\lambda_{k} M_{k}=\left\{\sum_{i=1}^{k} \lambda_{i} x_{i}: x_{i} \in M i, i=1, \ldots, k\right\}$

is convex.

- Taking the image under affine mapping: if $M \subset \mathbf{R}^{\mathrm{n}}$ is convex and $\mathrm{x} \mapsto \mathrm{A}(\mathrm{x}) \equiv \mathrm{Ax}+\mathrm{b}$ is an affine mapping from $\mathbf{R}^{\mathrm{n}}$ into $\mathbf{R}^{\mathrm{m}}$ (A is $\mathrm{m} \times \mathrm{n}$ matrix, b is m-dimensional vector), then the set

$A(M)=\{y=A(x) \equiv A x+b \mid x \in M\}$

is a convex set in $\mathbf{R}^{\mathrm{m}}$;

- Taking the inverse image under affine mapping: if $M \subset \mathbf{R}^{\mathrm{n}}$ is convex and y $\mathrm{x} \mapsto \mathrm{Ay}+\mathrm{b}$ is an affine mapping from $\mathbf{R}^{\mathrm{m}}$ to $\mathbf{R}^{\mathrm{n}}$ ( $\mathrm{A}$ is $\mathrm{n} \times \mathrm{m}$ matrix, $\mathrm{b}$ is $\mathrm{n}$-dimensional vector),then the set $A(M)=\{y=A(x) \equiv A x+b \mid x \in M\}$ is a convex set in $\mathbf{R}^{m}$.

\section{TOPOLOGICAL PROPERTIES OF CONVEX SETS}

Convex sets and closely related objects - convex functions - play the central role in Optimization. To play this role properly, the convexity alone is insufficient; we need convexity plus closedness. From the analysis we already know about the most basic topology-related notions - convergence of sequences of vectors, closed and open sets in $\mathbf{R}^{n}$. Here are three more notions we will use: The closure. It is clear from definition of a closed set that the intersection of any familyof closed sets in $\mathbf{R}^{n}$ is also closed. From this fact it, as always, follows that for any subset $M$ of $\mathbf{R} n$ there exists the smallest closed set containing $M$; this set is called the closure of $M$ and is denoted $\operatorname{cl} M$. In Analysis they prove the following inner description of the closure of a set in a metric space 
ISSN: 2362-1303 (Paper) | eISSN: 2362-1311 (Online)

JOURNAL OF ADVANCED ACADEMIC RESEARCH (JAAR)

(and, in particular, in $\mathbf{R}^{n}$ ):

The closure of a set $\mathbf{M} \subset \mathbf{R}^{\mathrm{n}}$ is exactly the set comprised of the limits of all converging sequences of elements of $\mathrm{M}$.

The interior.Let $M \subset R^{n}$. We say that a point $x \in M$ is an interior for $M$, if some

neighborhood of the point is contained in $M$, i.e., there exists centered at $x$ ball of positive radius which belongs to $M$ :

$\exists r>0 B_{r}(x) \equiv\{y|| y-x \mid \leq r\} \subset M$.

The set of all interior points of $M$ is called the interior of $M$ [notation: int $M$ ].

E.g.,

- The interior of an open set is the set itself;

- The interior of the closed ball $\{x|| x-a \mid \leq r\}$ is the open ball $\{x|| x-a \mid<r\}$

- The interior of a polyhedral set $\{x \mid A x \leq b\}$ with matrix $A$ not containing zero rows

is the set $\{x \mid A x<b\}$

Relative interior: Let $M \subset R^{n}$. We say that a point $x \in M$ is relative interior for $M$, if $M$ contains the intersection of a small enough ball centered at $\mathrm{x}$ with Aff (M): $\exists r>0 \operatorname{Br}(\mathrm{x}) \cap \operatorname{Aff}$ $(M) \equiv\{y|y \in \operatorname{Aff}(M)| y-x \mid, \leq r\} \subset M$. The set of all relative interior points of $M$ is called its relative interior [notation: ri $\mathrm{M}$

CONNECTED SETS :( https://en.wikibooks.org/wiki/Real_Analysis/Connected_Sets) / A set $\mathrm{A}$ in $R^{n}$ is connected if it is not a subset of the disjoint union of two open sets, both of which it intersects.

Examples: The set [0,2] cannot be covered by two open, disjoint intervals; for example, the open sets $(-1,1)$ and $(1,2)$ do not cover $(0,2)$ because the point $\mathrm{x}=1$ is not in their union. Thus $(0,2)$ is connected.

However, the set $\{0,2\}$ can be covered by the union of $(-1,1)$ and $(1,3)$, so $\{0,2\}$ is not connected. Definition: A set is path-connected if any two points can be connected with a path without exiting the set.

A useful example is $R^{2} \backslash\{(0,0)\}$. Any two points a and b can be connected by simply drawing a path that goes around the origin instead of right through it; thus this set is path-connected.

However, $R \backslash\{0\}$ is not path-connected, because for $\mathrm{a}=-3$ and $\mathrm{b}=3$, there is no path to connect a and $\mathrm{b}$ without going through $\mathrm{x}=0$.

As should be obvious at this point, in the real line regular connectedness and path-connectedness are equivalent; however, this does not hold true for $R^{n} R^{n}$ with $\mathrm{n}>1$. When this does not hold, path-connectivity implies connectivity; that is, every path-connected set is connected.

Definition: A set A is simply-connected if any loop completely contained in A can be shrunk down to a point without leaving A.

An example of a Simply-Connected set is any open ball in $R^{n} R^{n}$

Vol. 6. No. I

www.phdcentre.edu.np 
. However, the previous path-connected set $R^{2} \backslash\{(0,0)\}$ is not simply connected, because for any loop $\mathrm{p}$ around the origin, if we shrink $\mathrm{p}$ down to a single point we have to leave the set at $(0,0)$.

\section{CONNECTED SPACE}

Definition: A topological space $X$ is said to be disconnected if it is the union of two disjoint non-empty open sets. Otherwise, $X$ is said to be connected. A subset of a topological space is said to be connected if it is connected under its subspace topology. Some authors exclude the empty set (with its unique topology) as a connected space.

A space in which all components are one-point sets is called totally disconnected Related to this property, a space $X$ is called totally separated if, for any two distinct elements $x$ and $y$ of $X$, there exist disjoint open sets $U$ containing $x$ and $V$ containing $y$ such that $X$ is the union of $U$ and $V$. Clearly, any totally separated space is totally disconnected, but the converse does not hold.

\section{Path Connectedness}

Definition: A path-connected space is a stronger notion of connectedness, requiring the structure of a path. A path from a point $x$ to a point $y$ in a topological space $X$ is a continuous function $f$ from the unit interval $[0,1]$ to $X$ with $f(0)=x$ and $f(1)=y$. A path-component of $X$ is an equivalence class of $X$ under the equivalence relation which makes $x$ equivalent to $y$ if there is a path from $x$ to $y$. The space $X$ is said to be path-connected (or pathwise connected or 0connected) if there is exactly one path-component, i.e. if there is a path joining any two points in $X$. Again, many authors exclude the empty space.

Every path-connected space is connected. But the converse is not always true: examples of connected spaces that are not path-connected include the extended long line $L^{*}$ and the topologist's sine curve.

Subsets of the real line $\mathbf{R}$ are connected if and only if they are path-connected; these subsets are the intervals of $\mathbf{R}$. Also, open subsets of $\mathbf{R}^{n}$ or $\mathbf{C}^{n}$ are connected if and only if they are path-connected. Additionally, connectedness and path-connectedness are the same for finite topological spaces.

\section{Arc connectedness}

A space $X$ is said to be arc-connected or arcwise connected if any two distinct points can be joined by an $\operatorname{arc}$, that is a path $f$ which is a homeomorphism between the unit interval $[0,1]$ and its image $f([0,1])$. It can be shown any Hausdorff space which is path-connected is also arcconnected.

An example of a space which is path-connected but not arc-connected is provided by adding a second copy 0 ' of 0 to the nonnegative real numbers $[0, \infty)$. One endows this set with a partial order by specifying that $0^{\prime}<a$ for any positive number $a$, but leaving 0 and $0^{\prime}$ incomparable. One then endows this set with the order topology, that is, one takes the open intervals $(a, b)=\{x \mid a<x<b\}$ and the half-open intervals $[0, a)=\{x \mid 0 \leq \mathrm{x}<a\},\left[0^{\prime}, a\right)=\{x \mid$ $\left.0^{\prime} \leq x<a\right\}$ as a basefor the topology. The resulting space is a $\mathrm{T}_{1}$ space but not a Hausdorff space. Clearly 0 and 0 ' can be connected by a path but not by an arc in this space. 
ISSN: 2362-1303 (Paper) | eISSN: 2362-1311 (Online)

JOURNAL OF ADVANCED ACADEMIC RESEARCH (JAAR)

June 2019

\section{Locally connectedness}

A topological space is said to be locally connected at a point $x$ if every neighborhood of $x$ contains a connected open neighborhood. It is locally connected if it has a base of connected sets. It can be shown that a space $X$ is locally connected if and only if every component of every open set of $X$ is open.

Similarly, a topological space is said to be locally path-connected if it has a base of path-connected sets. An open subset of a locally path-connected space is connected if and only if it is path-connected. This generalizes the earlier statement about $\mathbf{R}^{n}$ and $\mathbf{C}^{n}$, each of which is locally path-connected. More generally, any topological manifold is locally path-connected. Locally connected does not imply connected, nor does locally path-connected imply path connected. A simple example of a locally connected (and locally path-connected) space that is not connected (or path-connected) is the union of two separated intervals in $R$, such as $(0,1)$ and $(2,3)$.

A classical example of a connected space that is not locally connected is the socalled topologist's sine curve, defined as $K=\{(0,0)\} \cup\left\{x, \sin \left(\frac{1}{x}\right): x \in(0,1]\right\}$, with the Euclidean topology induced by inclusion in $\mathrm{R}^{2}$.

\section{ANALYSIS OF THEOREMS}

\section{Theorem 1: Every convex set in $\mathrm{R}^{\mathrm{k}}$ is connected.(}

Proof: We prove this by contradiction in this. So we suppose the set $\mathrm{E}$ is convex and yet not connected, and so equals the union of two disjoint, non-empty open sets A and B. Since E is convex, for any $x \in A, y \in B$, there exists a mapping $f$ such that

$$
f(\lambda)=\lambda x+(1-\lambda) y
$$

for all $\lambda \in(0,1)$. This mapping is continuous and one-to-one, hence its image, which we call call $\mathrm{P}$ for "path", is both connected and open since $(0,1)$ is both connected and open. Since A and $\mathrm{B}$ are disjoint, it follows $\mathrm{P} \cap \mathrm{A}$ and $\mathrm{P} \cap \mathrm{B}$ are also disjoint. $\mathrm{P} \cap \mathrm{A}$ and $\mathrm{P} \cap \mathrm{B}$ are non-empty because $\mathrm{x}$ and $\mathrm{y}$ are taken from open sets and so therefore have a "buffer zone" of points around them which $\mathrm{P}$ will have to intersect. They are also open sets since $\mathrm{P}$ is open and so are $\mathrm{A}$ and $\mathrm{B}$. Hence $\mathrm{P} \cap \mathrm{A}$ and $\mathrm{P} \cap \mathrm{B}$ are two disjoint, non-empty open sets whose union is $\mathrm{P}$. Therefore $\mathrm{P}$ must be disconnected which contradicts that it is connected since it is a continuous image of a connected set.

Theorem 2: Every convex set is connected, but the converse is not true. Example: The ring region $K=\left\{(x, y), 1<x^{2}+y^{2}<9\right\}$ in the plane is connected but not convex.

Proof: The points $\mathrm{A}(2,0)$ and $\mathrm{B}(-2,0)$ belong to $\mathrm{K}$, but the line segment $\mathrm{AB}$ doesn't belong to $\mathrm{K}$, hence non-convex region. $\mathrm{K}$ is pathwise connected and connected since for any points $\mathrm{A}$ and B.K can be joined with a path.

Theorem 3: A convex connected set is simply connected but not vice versa 
ISSN: 2362-1303 (Paper) | eISSN: 2362-1311 (Online)

JOURNAL OF ADVANCED ACADEMIC RESEARCH (JAAR)

Proof: Convex set is simply connected (even contractible). But what makes we think that the converse holds? For example let $\mathrm{V}$ be a real normed vector space and for any $v, w \in V$ consider

$$
[v, w]=\{x \in V \mid x=t v+(1-t) w \text { forsome } t \in[0,1]\}
$$

i.e. the line segment between $\mathrm{v}$ and $\mathrm{w}$. With that the definition of convexity is quite simple: $A \subseteq V$ is convex iff $[v, w] \subseteq A$ for any $v, w \in A$

Theorem 4: Let Abe a star convex subset of a vector space V over R or C.

Then A is path-connected.

Proof

Let $x_{1}, x_{2} \in A$.

Let $\mathrm{a} \in \mathrm{A}$ be a star center of $\mathrm{A}$.

By definition of star convex set, it follows that for all $t \in[0 . .1]$ we have $t x_{1}+(1-t) a, t x_{2}+(1-t) a \in A$.

Define two paths $\gamma_{1}, \gamma_{2}: t \in[0 . .1] \rightarrow A$ by $\gamma_{1}(t)=t x_{1}+(1-t) a$ and $\gamma_{2}(t)=t a+(1-t) x_{2}$ $\mathrm{As}_{2}(t)=(1-t) x_{2}+(1-(1-t)) a$, and $(1-t) \in[0 . .1](1-t) \in[0 . .1]$, it follows that $\gamma_{2}(t) \in A$.

Note that $\gamma_{1}(0)=x_{1}, \gamma_{1}(1)=\gamma_{2}(0)=a$, and $\gamma_{2}(1)=x_{2}$.

Define $\gamma:[0 . .1] \rightarrow A$ as the concatenation $\gamma_{1} ; \gamma_{2}$.

Then $\gamma$ is a path in A joining $x_{1}$ and $x_{2}$, so A is path-connected.

Theorem 5: Star Convex Set is Path-Connected

Theorem 6: Hollow bodies (Sphere, torus) are connected but nut convex.

\section{CONCLUSIONS}

From theorems and literatures mentioned above we can say that all convex sets are connected but all connected sets are not convex. So, convexity cannot be replaced with the connectedness of C.

\section{REFERENCE}

Morris, Carla C. and Stark, Robert M., Finite mathematics: Models and applications. John Wiley \& Sons. p. 121. ISBN 9781119015383. Retrieved 5 April 2017.

Kjeldsen, Tinne, H. (2010) "History of convexity and mathematical programming" (PDF). Proceedings of the International Congress of Mathematicians, 3233-3257. doi:10.1142/9789814324359_0187. Retrieved 5 April 2017.

Bachem, Achimand, K. Walter. Linear programming duality: An introduction to oriented matroids. Springer Science \& Business Media. p. 13. ISBN 9783642581526. Retrieved 5 April 2017.

Pao, H.-K., Geiger, D. and Rubin, N. (1999). "Measuring convexity for figure/ground separation “.In 7th IEEE International Conference on Computer Vision.

Preparata, F., and Shamos, I. (1985). Computational geometry: An introduction (2nd ed.). New York:Springer-Verlag.

Vol. 6. No. I

www.phdcentre.edu.np 
ISSN: 2362-1303 (Paper) | eISSN: 2362-1311 (Online)

Rosin, P. L. (2000). "Shape partitioning by convexity". IEEE Transactions on Systems Man and Cybernetics Part, 30, 202-210.

https://math.stackexchange.com/questions/2167325/prove-every-convex-set-in-mathbbrk-isconnected/2167343 )

https://www.researchgate.net/post/Convex_set_and_connected_set

https://math.stackexchange.com/questions/2167325/prove-every-convex-set-in-mathbbrk-isconnected/2167343 )

https://proofwiki.org/wiki/Star_Convex_Set_is_Path-Connected 